\title{
Biomarker Testing to Estimate Under-Reported Heavy Alcohol Consumption by Persons with HIV Initiating ART in Uganda
}

\author{
Judith A. Hahn • Mwebesa B. Bwana • \\ Martin A. Javors · Jeffrey N. Martin • \\ Nneka I. Emenyonu • David R. Bangsberg
}

Published online: 10 August 2010

(C) The Author(s) 2010. This article is published with open access at Springerlink.com

\begin{abstract}
Alcohol affects the transmission and treatment of HIV, yet may be under-reported in resource-limited settings. We compared self-reported alcohol consumption with levels of plasma carbohydrate-deficient transferrin $(\% \mathrm{CDT})$, a biomarker of heavy alcohol consumption, in persons initiating antiretroviral therapy in Uganda. Almost seven percent $(6.7 \%)$ of persons reporting abstaining and $10 \%$ reporting consuming 1-40 drinks in the prior month tested positive for \%CDT, and actual under-report may be higher due to low sensitivity of \%CDT. These results suggest likely under-report in those reporting abstaining and current drinking. Improved identification of heavy alcohol consumption is needed for research and clinical purposes.
\end{abstract}

Keywords Carbohydrate-deficient transferrin .

Biological markers - Alcohol drinking · HIV .

Antiretroviral therapy · Uganda

J. A. Hahn $(\bowtie)$. J. N. Martin

University of California, Box 0811, San Francisco,

CA 94143-0811, USA

e-mail: judy.hahn@ucsf.edu

M. B. Bwana · D. R. Bangsberg

Mbarara University of Science and Technology,

Mbarara, Uganda

M. A. Javors

University of Texas Health Science Center,

San Antonio, TX, USA

N. I. Emenyonu · D. R. Bangsberg

Massachusetts General Hospital, Boston, MA, USA

\section{Introduction}

Alcohol consumption has a substantial harmful impact on the HIV epidemic in sub-Saharan Africa. Alcohol consumption has been a consistent risk factor for HIV acquisition in this region [1] and is associated with decreased antiretroviral therapy (ART) adherence worldwide [2]. In addition, many authors have hypothesized a link between alcohol consumption and HIV disease progression [3].

Uganda has an HIV prevalence of $5.4 \%$ and one of the highest per capita volumes of consumption of alcohol in the world. The per capita adult alcohol consumption is estimated to be 18.61 of pure alcohol per year [4]. Almost one quarter $(23.8 \%)$ of men and $7.0 \%$ of women report typically drinking five or more drinks per drinking occasion at least once a month in the prior year [4]. However, self-reported recent heavy alcohol consumption in a sample of HIV-positive persons initiating ART in Uganda was quite low, with only $3 \%$ reporting typically drinking six or more drinks per drinking occasion at least once a month in the prior year [5]. If alcohol consumption is under-reported in the setting of HIV, then the true magnitude of the effect of alcohol on the HIV epidemic in sub-Saharan Africa will be undetected or under-estimated. In addition, clinicians need to know about their patients' alcohol consumption in order to assess potential barriers to ART adherence.

Therefore, it is important to accurately assess the level of alcohol consumption among HIV positive patients. The goal of this study was to use a highly specific biochemical marker of heavy alcohol consumption, carbohydrate-deficient transferrin (CDT), to estimate the level of underreported heavy alcohol consumption among persons initiating ART in Uganda. 


\section{Methods}

\section{Study Subjects}

From 2005 through 2009, 500 HIV-infected adults initiating ART were recruited from the Mbarara Regional Referral Hospital Immune Suppression Syndrome (ISS) Clinic in southwestern Uganda for a cohort study of HIV treatment outcomes. Eligibility criteria included being at least age 18 , being willing to have serial blood draws and at-home ART adherence monitoring, and having received a prescription to initiate ART in the next week. For this analysis, we conducted CDT testing on stored plasma for all subjects in the cohort who reported any alcohol consumption in the prior year at baseline (37\%) and for whom there was sufficient archival plasma (61\%). We additionally selected a random sample of 54 subjects who reported no alcohol consumption in the prior year $(28 \%$ of those with sufficient plasma).

All procedures were approved by the Institutional Review Boards of the University of California, San Francisco, Partners HealthCare, and the Mbarara University of Science and Technology.

\section{Alcohol Assessment}

Alcohol consumption was reported at baseline using the World Health Organization Alcohol Use Disorders Identification Test (AUDIT), in addition to a question about when, if ever, alcohol was last consumed; the number of days in the prior 30 that alcohol was consumed; the typical number of standard drinks (14 grams of pure alcohol) consumed per drinking day; and the total amount of money spent on alcohol in the prior 30 days. The number of drinks per drinking day was missing for those who reported solely drinking beverages served in containers from which the number of drinks could not be estimated ( $n=2,1 \%$ of the sample). Other missing values are noted in the table.

\section{Carbohydrate-Deficient Transferrin (CDT)}

Biochemical markers of alcohol consumption have shown promise as objective measures of alcohol consumption. CDT is an abnormal transferrin profile that is caused by sustained heavy drinking [6]. While the results have varied, CDT is typically elevated after $50-80 \mathrm{~g}$ of ethanol (i.e., 3.5-6 US standard drink units) consumed daily or almost daily over 14 days [7], with all but the heaviest drinkers returning to normal after four weeks of abstinence [8]. Disialotransferrin is one of the minor glycoforms of human transferrin, and the percentage of total CDT that is disialotransferrin, as determined by high-performance liquid chromatography (HPLC), is the recommended method for measuring \%CDT [9]. The percent of abstainers and moderate drinkers combined that tested \%CDT positive (i.e., the percent false positive, or one minus the specificity of the test) using a cutoff of $1.8 \%$ was $0 \%$ in Italy [10] and Sweden [9] and 5.6\% in a multi-national study [6]. In the latter study, the specificity was $100 \%$ when moderate drinkers were excluded, and the authors noted that errors in self-report were likely among the moderate drinkers [6]. The sensitivity of \%CDT for detecting heavy drinking at the $1.8 \%$ cutoff in that study was $44.6 \%$. Despite this low sensitivity, we chose \%CDT because of its high specificity as well as its ability to be quantified in plasma. Other candidate biomarkers include phosphatidylethanol, which is highly sensitive and specific but currently only available for testing in whole blood [11], and traditional markers such as $\gamma$-glutamyl transpeptidase and mean corpuscular volume which are widely available but also lack sensitivity [11], and are subject to alterations due to HIV disease and ART use.

\section{Laboratory Methods}

Plasma was stored at $-70^{\circ} \mathrm{C}$ at the Makerere UniversityJohns Hopkins University Research Collaboration Laboratories and shipped on dry ice to the AIDS Specimen Bank in San Francisco. We (Javors) conducted HPLC to detect $\% \mathrm{CDT}$ in stored plasma samples using the methods as described by Helander et al. [9].

\section{Statistical Analysis}

We report the percent of participants with positive \%CDT tests (and exact binomial 95\% confidence intervals) by the duration since last consumed alcohol, and among those reporting drinking alcohol in the prior 30 days, by the following measures of drinking in the prior 30 days: the number of days drinking, the typical number of drinks per day, the total number of drinks, and the total amount of money spent on alcohol. The total number of drinks was calculated by multiplying the number of days drinking by the typical number of drinks per day. We divided these variables into heavy and not heavy categories at their 75 th percentile values as follows: drinking more than 15 days in the prior month (yes/no), typically drinking more than four drinks per occasion (yes/no), drinking more than 40 drinks in the past month (yes/no), and spending 15,000 Uganda Shillings (USH, 1 US dollar equaled approximately 1,600 USH during the study period) or more on alcohol in the prior month (yes/no). We calculated exact $95 \%$ confidence intervals for the proportion \%CDT positive and conducted Fisher exact tests to determine whether the proportion 
$\%$ CDT positive was associated with the drinking variables. We performed the Breslow-Day test of homogeneity to test for interaction by gender because previous research has shown lower sensitivity for \%CDT among females [6], and concluded there was interaction if $P<0.15$.

\section{Results}

We tested plasma for 79 male $(48.5 \%)$ and 84 female $(51.5 \%)$ participants, with median age 36 (range 20-66). Overall, $6.7 \%(2.9-12.7 \%)$ of persons who reported abstaining for 30 days or more tested \%CDT positive (Table 1). We divided the percent of \%CDT positive results by the reported sensitivity of the test $(44.6 \%)$ to back-calculate the percent of those reporting abstaining who likely consumed heavy alcohol; this estimate is $15.0 \%$.

Of the 43 who reported any alcohol consumption in the prior 30 days, 7 (16.3\%; 6.8-30.7\%) tested positive. Among current drinkers, there were significant associations with \%CDT and the number of days reported drinking, the total number of drinks, and the amount of money spent drinking (all Fisher's exact test $P<0.05$ ), and a borderline significant association with number of drinks per day (Fisher's exact test $P=0.09$ ). There was no significant interaction by sex (all Breslow-Day $P>0.15$ ).

Table 1 Carbohydrate-deficient transferrin (\%CDT) positive by selfreported date of last consumption and measures of recent alcohol consumption

$n \% \mathrm{CDT}$ positive/ $\mathrm{N}$

$(\% ; 95 \%$ exact binomial $\mathrm{CI})$

When last consumed alcohol

$\begin{array}{lc}\text { More than } 30 \text { days ago } & 8 / 120(6.7 \% ; 2.9-12.7 \%) \\ \text { Within the past } 30 \text { days } & 7 / 43(16.3 \% ; 6.8-30.7 \%) \\ \text { Prior } 30 \text { days alcohol consumption } & \\ \text { Number of days drinking }(n=2 \text { missing) } \\ \begin{array}{ll}1-15 & 3 / 31(9.7 \% ; 2.0-25.8 \%) \\ 16-30 & 4 / 10(40.0 \% ; 12.2-73.4 \%)\end{array}\end{array}$

Typical number of drinks per drinking day ( $n=2$ missing, $n=2$ drank home brewed/distilled only)

$$
\text { 1-4 4/32 (12.5\%; 3.5-28.9\%) }
$$$$
\geq 5 \quad 3 / 7(42.9 \% ; 9.9-81.6 \%)
$$

Total number of drinks ( $n=4$ missing)

$$
\begin{array}{ll}
1-40 & 3 / 30(10.0 \% ; 2.1-26.5 \%) \\
\geq 41 & 4 / 9(44.4 \% ; 13.7-78.8 \%)
\end{array}
$$

Total amount of money spent on alcohol ( $n=3$ missing)

$$
\begin{array}{ll}
<15,000 \mathrm{USH} & 2 / 30(6.7 \% ; 1.0-22.1 \%) \\
\geq 15,000 \mathrm{USH} & 4 / 10(40.0 \% ; 12.2-73.4 \%)
\end{array}
$$

\section{Discussion}

We used \%CDT, a highly specific marker of heavy alcohol consumption (i.e., one that would result in very few false positives) and found that approximately $7 \%$ of self-reported abstainers were positive. Using the published sensitivity of \%CDT, we estimate that the actual percent of those reporting abstaining who consumed heavy alcohol was $15 \%$.

Self-reported heavy alcohol consumption was confirmed by positive \%CDT in a high proportion of persons reporting drinking frequently and/or a large number of drinks. However, a substantial fraction of those reporting few drinking days, light consumption on drinking days, or low amounts of money spent on alcohol were also \%CDT positive, indicating that there are challenges to accurate report of the level of drinking even among those willing to report recent alcohol consumption.

Lower than expected self-reported alcohol consumption in persons with HIV in Uganda may occur for several reasons. First, standard measures of alcohol consumption are based on reporting the frequency of consuming standard drink units which are problematic because neither drink size nor alcohol content is standard in many places [12], including Uganda. While commercially prepared alcohol is available, much of the available alcohol is brewed or distilled at home or locally, with variable alcohol concentrations. In addition, home or locally brewed or distilled forms of alcohol can be obtained in varying container sizes and may be consumed using long wooden or plastic straws shared among several people at the pot side, making it difficult to quantify consumption.

Second, social desirability bias may also cause underreporting if there is the perception of negative consequences associated with reported use. Persons infected with HIV may under-report their alcohol consumption if they fear that they will be denied ART. HIV-infected patients in Uganda, especially those initiating ART, are counseled to abstain from drinking by clinic counselors.

Our study was limited by a modest sample size. In addition, the lack of sensitivity of \%CDT, the best commercially available biomarker for testing of plasma, constrained the interpretation of the estimates. There is a small chance of false positives, which would bias our estimates upwards. In order to counteract this, we considered using a higher cutoff value for \%CDT positive. However, increasing the \%CDT cutoff from 1.8 to $2.0 \%$ resulted in only a $1.5 \%$ gain in specificity accompanied by a $8.5 \%$ decline in sensitivity in a recent study [6]. We recommend that future studies collect whole blood in order take advantage of the high sensitivity and specificity of phosphatidylethanol as a marker of heavy alcohol consumption [11]. 
Improved measurement of alcohol exposure is important to understand the effect of alcohol on HIV outcomes. For example, studies of the effect of alcohol on HIV disease progression have had mixed results [3], possibly due to difficulties in measuring alcohol consumption. In addition, clinicians treating HIV need to be able to assess the level of alcohol consumption in their patients in order to consider potential barriers to adherence. The implications of this study are that researchers and clinicians should be aware that a non-negligible proportion of persons with HIV in Uganda under-report heavy alcohol consumption. This study shows that heavy alcohol consumption is underreported via denial of any alcohol consumption and underreport of the quantity of alcohol consumed. Under-report can only be addressed by developing and implementing sensitive and specific biologic markers of alcohol consumption or devising methods that promote alcohol consumption disclosure.

Acknowledgment This study was supported by NIH R01 MH54907 and Hellman Family Award for Early Career Investigator.

Open Access This article is distributed under the terms of the Creative Commons Attribution Noncommercial License which permits any noncommercial use, distribution, and reproduction in any medium, provided the original author(s) and source are credited.

\section{References}

1. Fisher JC, Bang H, Kapiga SH. The association between HIV infection and alcohol use: a systematic review and meta-analysis of African studies. Sex Transm Dis. 2007;34(11):856-63.
2. Hendershot CS, Stoner SA, Pantalone DW, Simoni JM. Alcohol use and antiretroviral adherence: review and meta-analysis. J Acquir Immune Defic Syndr. 2009;52(2):180-202.

3. Hahn JA, Samet JH. Alcohol and HIV disease progression: weighing the evidence. Curr HIV AIDS Rep. 2010 (in press).

4. Global Status Report on Alcohol. 2004. http://www.who.int/sub stance_abuse/publications/statusreportalcoholafro/en/index.html. Accessed 4 Nov 2004.

5. Hahn JA, Maier M, Byakika-Tusiime J, Oyugi JH, Bangsberg DR. Hepatotoxicity during nevirapine-based fixed-dose combination antiretroviral therapy in kampala, Uganda. J Int Assoc Physicians AIDS Care (Chic Ill). 2007;6(2):83-6.

6. Bergstrom JP, Helander A. Clinical characteristics of carbohydrate-deficient transferrin (\%disialotransferrin) measured by HPLC: sensitivity, specificity, gender effects, and relationship with other alcohol biomarkers. Alcohol Alcohol. 2008;43(4): 436-41.

7. Golka K, Wiese A. Carbohydrate-deficient transferrin (CDT)-a biomarker for long-term alcohol consumption. J Toxicol Environ Health. 2004;7(4):319-37.

8. Helander A, Carlsson S. Carbohydrate-deficient transferrin and gamma-glutamyl transferase levels during disulfiram therapy. Alcohol Clin Exp Res. 1996;20(7):1202-5.

9. Helander A, Husa A, Jeppsson JO. Improved HPLC method for carbohydrate-deficient transferrin in serum. Clin Chem. 2003;49(11):1881-90.

10. Bianchi V, Ivaldi A, Raspagni A, Arfini C, Vidali M. Use of carbohydrate-deficient transferrin (CDT) and a combination of GGT and CDT (GGT-CDT) to assess heavy alcohol consumption in traffic medicine. Alcohol Alcohol. 2010;45:247-51.

11. Hannuksela ML, Liisanantti MK, Nissinen AE, Savolainen MJ. Biochemical markers of alcoholism. Clin Chem Lab Med. 2007; 45(8):953-61.

12. Papas RK, Sidle JE, Wamalwa ES, et al. Estimating alcohol content of traditional brew in western Kenya using culturally relevant methods: the case for cost over volume. AIDS Behav. 2010;14(4):836-44. 